\title{
Pulmonale Hypertonie: mikroRNAs in Pathogenese, Diagnostik und Therapie
}

\author{
Pulmonary Hypertension: microRNAs in Pathogenesis, Diagnosis and Therapy
}

Autoren

Institut
L. C. Huber, C. Leuenberger, M. Kohler, M. Brock

Klinik für Pneumologie, Universitätsspital Zürich eingereicht $\quad 2.1 .2014$ akzeptiert nach Revision 14. 3.2014

Bibliografie

Dol http://dx.doi.org/ 10.1055/s-0034-1365456 Online-Publikation: 8.4.2014 Pneumologie 2014; 68: 386-393 (c) Georg Thieme Verlag KG Stuttgart · New York ISSN 0934-8387

Korrespondenzadresse PD Dr. med. Lars C. Huber Klinik für Pneumologie Universitätsspital Zürich Rämistrasse 100 8091 Zürich Schweiz

lars.huber@usz.ch

\section{Zusammenfassung \\ $\nabla$}

Im Unterschied zur pulmonal-arteriellen Hypertonie, welche eine seltene Erkrankung ist, umfasst der Überbegriff der pulmonalen Hypertonie ein klinisch häufigeres Krankheitsbild. Die pathophysiologische Trias von Vasokonstriktion, Mikrothrombosen und Gefäßumbau (Remodelling) findet sich dabei unabhängig von der zugrundeliegenden Entität in unterschiedlich starker Ausprägung bei praktisch allen Formen der pulmonalen Hypertonie. In dieser Übersichtsarbeit werden neue Aspekte in der Pathogenese des pulmonalvaskulären Remodellings, insbesondere mikroRNA-abhängige Mechanismen, besprochen.

MikroRNAs sind kleine RNA-Fragmente, welche an die messengerRNA eines Zielgenes binden, was zum Abbau des Zielgenes oder zur Translationshemmung des betreffenden Gens führt („gene silencing“). Im Zusammenhang mit dem Gefäßumbau bei der pulmonalen Hypertonie ist vor allem die Assoziation von mikroRNAs und des Bone Morphogenetic Protein Rezeptor Typ II interessant: In verschiedenen Formen der pulmonalen Hypertonie wurde eine reduzierte Expression von Bone Morphogenetic Protein Rezeptor Typ II auf Endothelien und Gefäßmuskelzellen gefunden. Durch spezifische Hemmung mittels AntagomiRs bieten mikroRNAs die Möglichkeit für einen potenziell kausalen Therapieansatz. MikroRNAs werden zudem als Biomarker im Serum für Diagnose, Schweregrad und Prognose der pulmonalen Hypertonie validiert.

\section{Definition}

$\nabla$

Eine pulmonale Hypertonie liegt dann vor, wenn der pulmonal-arterielle Mitteldruck (mPAP) $25 \mathrm{mmHg}$ übersteigt [1]. Definitionsgemäß wird die Diagnose der pulmonalen Hypertonie somit invasiv mittels Rechtsherzkatheter gestellt. Echo-

\section{Abstract \\ $\nabla$}

Whereas pulmonary arterial hypertension is an orphan disease, the term pulmonary hypertension includes several common entities and is of major clinical significance. The pathophysiological triad of vasoconstriction, microthrombosis and vascular remodeling is found in most forms of pulmonary hypertension, independently of the underlying etiology. In this review, novel aspects in the pathogenesis of the remodeling, in particular microRNAs, will be discussed. MicroRNAs are small RNA fragments which bind specifically to the mRNA of a target gene thus decreasing its stability or inhibiting further translation ("gene silencing"). Of major interest is the association between microRNAs and the expression of bone morphogenetic protein receptor type II which has been found to be dysregulated on pulmonary endothelial and vascular smooth muscle cells in several forms of pulmonary hypertension. The specific inhibition of microRNAs by antagomiRs makes microRNAs a potential therapeutic target. Moreover, microRNAs are being validated in serum as biomarkers for diagnosis, severity and prognosis of pulmonary hypertension. kardiografisch kann der mPAP zwar geschätzt bzw. aus dem systolischen Druck berechnet werden [2], aufgrund von Limitationen (Untersucherabhängigkeit, Schallqualität, Trikuspidalinsuffizienz) eignet sich die Echokardiografie aber vor allem zum Screening und als Verlaufsparameter. Andere Methoden, insbesondere die Bildgebung 
mittels Magnetresonanztomografie [3], werden bisher in der Klinik noch nicht routinemäßig angewendet.

Andere Kriterien neben dem mPAP, wie beispielsweise das Herzminutenvolumen, der Wedge-Druck (genauer der pulmonale kapilläre Verschlussdruck [PAOP]), der pulmonale vaskuläre Widerstand oder der transpulmonale Gradient (als Differenz zwischen dem pulmonalen Mitteldruck und dem kapillären Verschlussdruck), werden zur Diagnose nicht oder nur zur weiteren pathophysiologischen Einteilung der pulmonalen Drucksteigerung benötigt. Unterschieden wird des Weiteren eine präkapilläre pulmonale Hypertonie, bei der die Drucksteigerung in der Regel in den Widerstandsgefäßen vor dem kapillären Gefäßbett der Lunge stattfindet und mit einem niedrigen PAOP $(<15 \mathrm{mmHg})$ sowie mit einem erhöhten transpulmonalen Gradienten (TP $>12$ $\mathrm{mmHg}$ ) einhergeht, und eine postkapilläre pulmonale Hypertonie, bei der sich die Drucksteigerung vom linken Herzen quasi über die Drucksäulen des pulmonal-venösen Systems auf die rechtsventrikuläre Seite überträgt. Letztere geht mit einem erhöhten PAOP einher und wird je nach Verhalten des transpulmonalen Gradienten in eine passive ( $\mathrm{TP}<12 \mathrm{mmHg}$ ) oder in eine reaktive (TP $>12 \mathrm{mmHg}$ ) Form „out-of-proportion“ unterteilt $[4,5]$.

Klinisch werden nach der Einteilung der Weltgesundheitsorganisation (WHO) fünf verschiedene Gruppen der pulmonalen Hypertonie unterschieden: die pulmonal-arterielle Hypertonie (PAH, WHO Klasse 1), die pulmonale Hypertonie im Zusammenhang mit Erkrankungen des linken Herzens (WHO Klasse 2) bzw. der Lunge (WHO Klasse 3), die chronisch-thromboembolisch bedingte Drucksteigerung (WHO Klasse 4) sowie die pulmonale Hypertonie als Folge verschiedener Entitäten (WHO Klasse 5), welche sich nicht einer der anderen vier Klassen zuordnen lassen. Während die PAH mit einer Inzidenz von 1 auf 2-15 Millionen pro Einwohner und Jahr eine seltene Krankheit ist [6], repräsentiert der Überbegriff der pulmonalen Hypertonie ein klinisch häufigeres Krankheitsbild, wobei genaue epidemiologische Zahlen diesbezüglich fehlen.

\section{Pathogenese}

$\nabla$

Interessanterweise münden verschiedene klinische Formen der pulmonalen Hypertonie in eine gemeinsame pathogenetische Endstrecke (final common pathway), wobei dies sowohl pathologisch-anatomisch [7] wie auch molekular [8] zutrifft. Die pathogenetische Trias aus Vasokonstriktion, in situ Thrombosierung und Remodelling beeinflussen und bedingen sich gegenseitig, wobei die Vasokonstriktion wahrscheinlich einem eher frühen Krankheitsstadium entspricht. In der durch Hypoxie experimentell induzierten pulmonalen Hypertonie werden allerdings auch spezifische vaskuläre Veränderungen im Sinne eines Remodellings bereits nach wenigen Wochen beobachtet. Für den Krankheitsverlauf ist das Remodelling von besonderer Bedeutung, da Chronizität und Unheilbarkeit der Erkrankung hauptsächlich durch den pulmonalen Gefäßumbau bestimmt werden.

Auf histologischer Ebene zeigt sich das Remodelling als Verdickung der Intima und zwiebelschalenartigem Wachstum der Gefäßendothelien mit Ausbildung einer Neo-Intima und deutlicher Einengung des Gefäßlumens sowie als überschießende Proliferation der glatten Muskelzellen mit Ausbildung einer stark muskularisierten Media (Media-Hypertrophie) und Transmigration der Muskelzellen aus der Media in die Intima [9]. Diese Veränderungen werden in unterschiedlich starker Ausprägung bei allen Formen der pulmonalen Hypertonie gefunden [10]. Einzig plexiforme Läsionen, also septierte, netzartige Unterteilungen des Gefäßlumens, sind pathognomonisch für die PAH [11].

In den letzten Jahren sind verschiedene Faktoren als mögliche Trigger für den Gefäßumbau untersucht worden (zusammengefasst in [8-12]): inflammatorische Zytokine (Interleukin (IL-) 1 und $6[13,14]$ ) und Entzündungszellen (z.B. durch ein unterschiedliches mRNA-Profil der zirkulierenden B-Lymphozyten [15]), Chemokine (Fractalkine [16-19]), Transkriptionsfaktoren wie z.B. Nuclear Factor of Activated T-cells (NFAT [20]), die Inhibierung spannungsabhängiger Kalium-Kanäle durch Hypoxie [21], mitogen aktive Transmitter (Serotonin [22,23]) usw. Genetische und nicht-genetische Veränderungen des bone morphogenetic protein receptor type II (BMPR2) sind aber die wichtigsten Faktoren.

Der BMPR2 ist ein Protein aus der Transforming Growth-Factorbeta-Familie, welche Wachstum und Differenzierung von Zellen steuert. Der BMPR2 ist auf der Zelloberfläche von Endothelien und glatten Muskelzellen lokalisiert und, nach Bindung eines entsprechenden Liganden (i.e. BMP-2,-4,-9 welche parakrin von zirkulierenden Mono- und Lymphozyten oder autokrin von Gefäßendothelien produziert werden), phosphoryliert der BMPR2 einen Partnerrezeptor (BMPR1), was über verschiedene Smad-Transkriptionsfaktoren, inhibitors of differentiation und Zellzyklusproteine eine proliferationshemmende intrazelluläre Signalkette in Gang setzt [24].

Mutationen im BMPR2, der auf dem langen Arm des Chromosoms 2 kodiert wird, wurden im Zusammenhang mit der $\mathrm{PAH}$ erstmals 2000 beschrieben [25] und werden in ca. $80 \%$ der familiären sowie in bis zu $25 \%$ der idiopathischen PAH gefunden, weshalb diese Formen heute als hereditäre PAH bezeichnet werden [24]. Träger der Mutation weisen generell einen schwereren Krankheitsverlauf auf [26]. BMPR2-Mutationen sind auch bei der veno-okklusiven Form der PAH beschrieben worden [27]. Die genetischen Veränderungen des BMPR2 sind in die WHOEinteilung der pulmonalen Hypertonie aufgenommen worden, ein genetisches Screening wird im klinischen Setting aber nicht generell empfohlen.

Darüber hinaus sind nicht-genetische Dysregulationen im Sinne einer verminderten Expression auch in anderen Formen der pulmonalen Hypertonie beschrieben worden, so z. B. bei Patienten mit Linksherzinsuffizienz [28], bei kongenitalen Herzerkrankungen [29], in der Hypoxie-induzierten pulmonalen Hypertonie und in verschiedenen Tiermodellen [30,31]. Bis vor kurzem ist darüber debattiert worden, ob es sich bei diesen nicht-genetischen Veränderungen um rein deskriptive Phänomene ohne pathogenetische Konsequenzen handelt. Mittlerweile konnte gezeigt werden, dass ein funktionell intakter BMPR2-Signalweg für die strukturelle und pathophysiologische Integrität der Lungenstrombahn von entscheidender Bedeutung ist.

\section{mikroRNAs}

Als wichtige Regulatoren für die Expression des BMPR2 konnten in den letzten Jahren mikroRNAs (miR) identifiziert werden. Seit dem Erstbeschrieb einer möglichen pathogenetischen Assoziation von miR und der Entwicklung einer pulmonalen Hypertonie [32] sind mehrere wichtige Publikationen erschienen, welche die Bedeutung von miR, von BMPR2 $[33,34]$ und insbesondere von miR in der Regulation von BMPR2 im Zusammenhang mit dem pulmovaskulären Remodelling untermauert haben. 


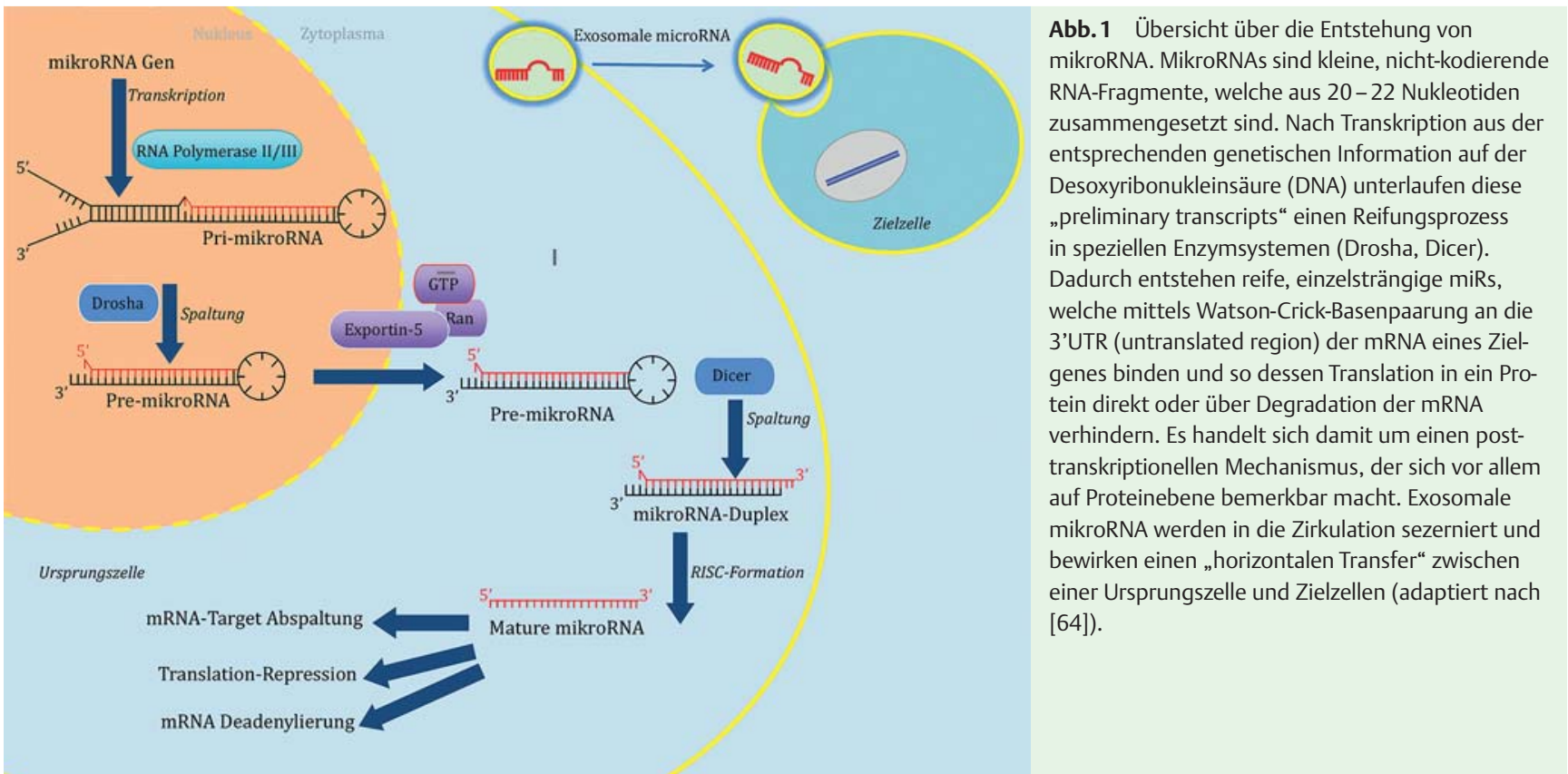

MiR sind kleine, nicht-kodierende RNA-Fragmente, welche aus 20-22 Nukleotiden zusammengesetzt sind. Nach Transkription aus der entsprechenden genetischen Information auf der Desoxyribonukleinsäure (DNA) unterlaufen diese „preliminary transcripts“ einen Reifungsprozess in speziellen Enzymsystemen (z. B. Drosha, Dicer, wobei letzteres bei der Hypoxie-induzierten pulmonalen Hypertonie ebenfalls reduziert exprimiert gefunden wurde [35]). Dadurch entstehen reife, einzelsträngige miR, welche mittels Watson-Crick-Basenpaarung an die 3'UTR (untranslated region) der mRNA eines Zielgenes binden und so dessen Translation in ein Protein direkt oder über Degradation der mRNA verhindern. Es handelt sich damit um einen posttranskriptionellen Mechanismus, der sich vor allem auf Proteinebene bemerkbar macht. Vereinfacht gesagt, werden miR generell mit Prozessen assoziiert, welche zu einer verminderten Genexpression (gene silencing) führen ( $\bullet$ Abb.1). Über hemmende Transkriptionsfaktoren kann dies natürlich auch zu einem positiven Feedback-Loop im Sinne von „Minus × Minus gibt Plus“ führen [36]. Die Bedeutung des zur entsprechenden miR komplementären „passenger-strands“ ist umstritten - er hat jedoch ein komplett anderes Spektrum an Zielgenen und spielt somit für ein bestimmtes Zielprotein einer miR bzw. für die Untersuchung einer bestimmten Krankheit keine Rolle. MiR werden zudem nicht nur innerhalb der Zelle gefunden, sondern können auch innerhalb von Exosomen sezerniert werden. Damit können miR auch „horizontal“ zwischen verschiedenen Zellen transferiert werden und (patho)physiologische Mechanismen unabhängig von der ursprünglichen Zelle beeinflusst werden [37,38]. Sekretorische miR sind zudem als Biomarker interessant.

Verschiedene miR binden an die 3'UTR der BMPR2-mRNA und führen so theoretisch zu einer verminderten Expression des BMPR2 auf Endothelien und glatten Gefäßmuskelzellen. Die Bedeutung dieser miR ist bereits untersucht worden oder ist Gegenstand laufender Forschungsarbeiten. Dabei geht es primordial um die Rolle von miR in der Pathogenese des pulmonalen Gefäßumbaus; andere Arbeiten fokussieren auf die Verwendung von miR als kausal-therapeutische Angriffspunkte. In der Folge werden die aktuellen Daten zu miR und pulmonaler Hypertonie vor- gestellt, eine Kurzübersicht gibt zudem $\bullet$ Tab.1, und die verschiedenen Signalwege sind in $\bullet$ Abb. 2 zusammengestellt.

\section{miR-17/miR-20}

Die Assoziation von miR-17/-20 und der Entwicklung einer pulmonalen Hypertonie wurde vor etwas mehr als vier Jahren postuliert [32].

miR-17 und miR-20 werden in einem cluster (miR-17/-92) kodiert, der mehrere miR umfasst. Dieser wird durch inflammatorische Zytokine, insbesondere das bei PAH-Patienten erhöht zirkulierende [13] und mit einer schlechteren Prognose [14] assoziierten IL-6 induziert. IL-6 agiert hauptsächlich über STAT3 (signal transducer and activator of transcription), seinen wichtigsten Transkriptionsfaktor, welcher wiederum an den Promoter von c13orf25 bindet. Diese phylogenetisch hochkonservierte Bindung führt zur Aufregulierung der reifen miR-17 und miR-20, welche konsekutiv an die mRNA von BMPR2 binden und so dessen Translation in das Protein verhindern. Dieser Mechanismus bietet in vitro eine erste Erklärung für die bis anhin nur rein deskriptiv festgestellte verminderte Expression von BMPR2 in verschiedenen Formen der pulmonalen Hypertonie. Das in plexiformen Läsionen nachgewiesene, konstitutiv aktive STAT3 [39] macht diese Hypothese zusätzlich attraktiv, da der Signalweg dann auch ohne erhöhten IL-6-Spiegel aktiv ist. Unterdessen wurde die wichtige Rolle von miR-17 und miR-20 wiederholt bestätigt: Caruso und Mitarbeiter erstellten ein miR-Profil während der Entwicklung einer experimentellen pulmonalen Hypertonie durch Hypoxie und Monocrotalin und fanden beide miR ebenfalls aufreguliert, wobei eine statistische Signifikanz nur im Monocrotalin-Modell gesehen wurde. Die Antagonisierung von miR-17 mittels intravenös applizierten AntagomiRs (siehe unten) korrigierte sowohl in der Hypoxie-induzierten pulmonalen Hypertonie wie auch im Monocrotalin-Model die BMPR2-Expression im Lungengewebe und führte zu einer deutlichen Verbesserung der rechtsventrikulären Hämodynamik (mPAP, rechter Ventrikel/rechtes Atrium) [40]. Auch die Hemmung der miR-20 - wel- 
Tab. 1 Überblick über die wichtigsten mikroRNAs im Kontext der pulmonalen Hypertonie (PH).

\begin{tabular}{|c|c|c|}
\hline mikroRNA & Effekt & Referenz \\
\hline miR-17p & $\begin{array}{l}\text { Aufreguliert in Hypoxie- und Monocrotalin-induzierter PAH. } \\
\text { Inhibierung begünstigt experimentelle PH. }\end{array}$ & Pullamsetti et al., 2012 [40] \\
\hline miR-20a & $\begin{array}{l}\text { BMPR2-Expression ist bei Inhibierung durch AntagomiR-20a reduziert. } \\
\text { Antagonisierung von miR-20a reduziert die experimentelle PH. }\end{array}$ & Brock et al., 2012 [41] \\
\hline miR-124 & $\begin{array}{l}\text { Vermindert in den glatten Muskelzellen der Media und in den Fibroblasten der Gefäßadventitia. } \\
\text { Steuert die perivaskuläre Inflammation. }\end{array}$ & $\begin{array}{l}\text { Kang et al., } 2013[44] \\
\text { Wang et al., } 2014[45]\end{array}$ \\
\hline miR-125a & $\begin{array}{l}\text { Im Serum von Patienten mit präkapillärer pulmonaler Hypertonie vermindert. } \\
\text { Potenzieller Biomarker. }\end{array}$ & Huber et al, 2013 [47] \\
\hline miR-130 & Erhöhte Zellproliferation über Inhibierung von CDKN1A. & Brock et al., 2013 [62] \\
\hline miR-145 & Deutlich erhöhte Spiegel bei PAH. Eine Antagonisierung scheint protektiv zu wirken. & Caruso et al., 2012 [49] \\
\hline miR-150 & $\begin{array}{l}\text { Im Serum von Patienten mit PAH vermindert vorhanden. Spiegel korrelieren mit Mortalität. } \\
\text { Potenzieller Biomarker. }\end{array}$ & Rhodes et al., 2012 [63] \\
\hline miR-204 & $\begin{array}{l}\text { Weniger exprimiert in Hypoxie- und Monocrotalin-induzierter PH. } \\
\text { Erhöhte Exprimierung von miR-204 reduziert die experimentell induzierte PH. }\end{array}$ & Courboulin et al., 2011 [51] \\
\hline miR-21 & $\begin{array}{l}\text { Überexprimiert in hypoxischer } \mathrm{PH} \text {. } \\
\text { Antagonisierung von miR-21 begünstigt die Entwicklung einer } \mathrm{PH} \text {. }\end{array}$ & Yang et al., 2012 [42] \\
\hline $\begin{array}{l}(\operatorname{miR}-22) \\
(\operatorname{miR}-30) \\
(\text { let-7) }\end{array}$ & $\begin{array}{l}\text { Alle signifikant vermindert vorhanden. Es zeigten sich allerdings deutliche Unterschiede } \\
\text { je nach Zeitpunkt und experimentellem Modell. }\end{array}$ & Caruso et al., 2010 [35] \\
\hline $\begin{array}{l}(\mathrm{miR}-322) \\
(\mathrm{miR}-451)\end{array}$ & $\begin{array}{l}\text { Beide deutlich verstärkt vorhanden. } \\
\text { Die miR-322 fiel nach } 21 \text { Tagen unter hypoxischen Konditionen bzw. nach Monocrotalin-Injektion } \\
\text { wieder auf das Level der Kontrollgruppe. }\end{array}$ & Caruso et al., 2010 [35] \\
\hline $\begin{array}{l}\text { miR-424 } \\
\text { miR-503 }\end{array}$ & $\begin{array}{l}\text { Vermindert vorhanden in PH. Interaktion mit Apelin-Signalweg. } \\
\text { Rescue-Expression verbessert das pulmonale Gefäßremodelling. }\end{array}$ & Kim et al., 2013 [53] \\
\hline
\end{tabular}

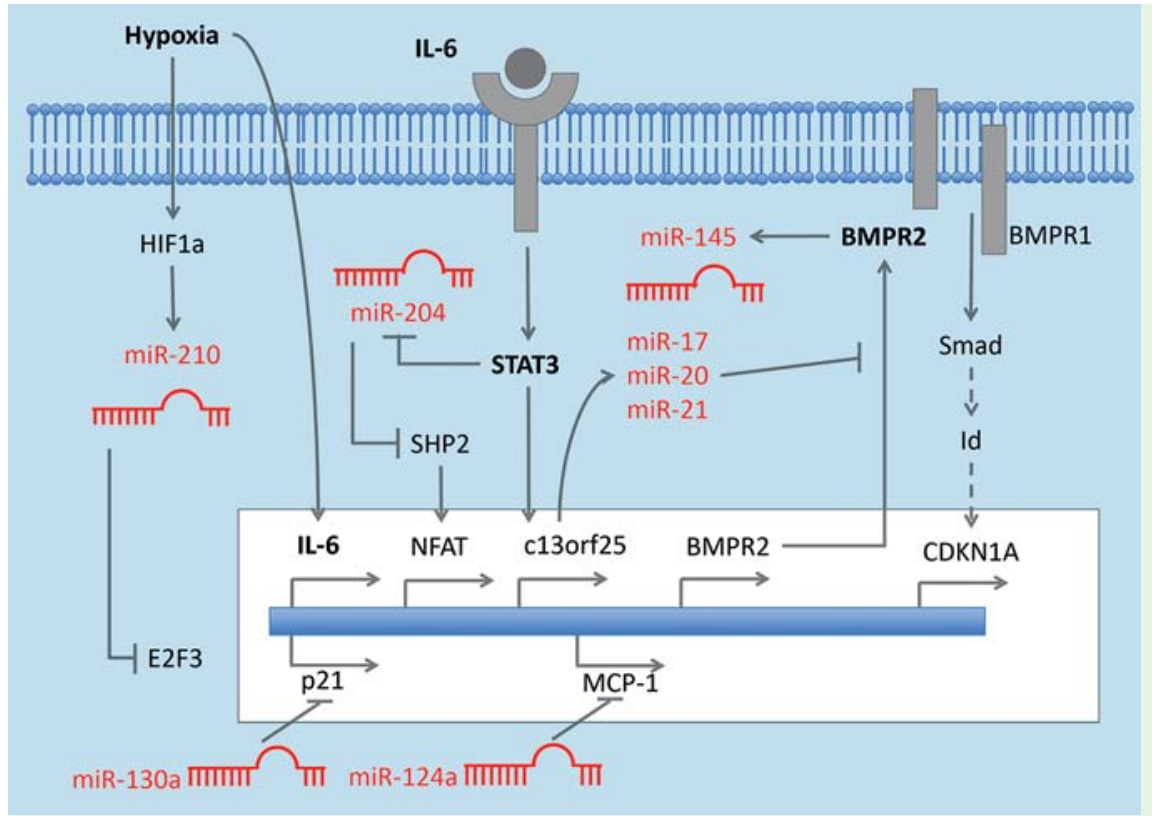

Abb.2 Übersicht über die Zielgene von mikroRNAs, die bei der Entstehung und morphologischen Fixierung von pulmonaler Hypertonie bekannt sind (Details siehe Tab. 1 und Text, ergänzt nach [65]). che sich strukturell nur durch 2 Nukleotide von der miR-17 unterscheidet, in vitro allerdings eindrücklichere Effekte auf den BMPR2 gezeigt hatte [32] - führte zu einer funktionellen Normalisierung des BMPR2 auf der Zelloberfläche, was in einem signifikant verminderten Gefäßremodelling der pulmonalen Arteriolen und einer geringeren rechtsventrikulären Hypertrophie resultierte [41]. Die Effekte auf den Gefäßumbau waren dabei am ehesten durch eine verminderte Proliferation der glatten Muskelzellen bedingt.
miR-21

$\nabla$

Die miR-21 kommt beim gesunden Menschen vor allem in distalen Lungenarteriolen sowie in der glatten Gefäßmuskulatur vor, was sie zu einem wichtigen Kandidaten im Kontext mit pulmonaler Hypertonie macht. Momentan ist die Rolle der miR-21 bei PAH kontrovers: Es gibt Daten, die zeigen erhöhte Levels von miR-21, andere zeigen eine deutliche Verminderung der miR-21 in PAH $[35,42,43]$. Wir favorisieren die Meinung, dass die miR-21, welche ebenfalls den BMPR2 antagonisiert, in PAH aufreguliert ist und so die Proliferation von pulmonalen Endothelien und Gefäßmuskelzellen erhöht. Eine Überexpression dieser miR führte ent- 
sprechend zu einer erhöhten Proliferation der human pulmonary artery smooth muscle cells. Zudem haben diverse andere Studien gezeigt, dass die miR-21 eine wichtige Rolle für den pulmonalen Gefäßumbau spielt [42].

\section{miR-124}

$\nabla$

Kürzlich publizierte Arbeiten haben gezeigt, dass die miR-124 in pulmonary artery smooth muscle cells [44] und in Fibroblasten der pulmonalen Gefäßadventitia reduziert vorhanden ist [45]. Interessanterweise ist die miR-124 auch bei verschiedenen malignen Tumoren vermindert exprimiert, was mit einem aktivierten Phänotyp und einer vermehrten Proliferation und Migration von Zellen in Verbindung gebracht worden ist. In Muskelzellen interagiert miR-124 mit dem Transkriptionsfaktor NFAT, der bereits mit anderen mikroRNAs und der Pathogenese der pulmonalen Hypertonie in Zusammenhang gebracht wurde (siehe miR-204). In Fibroblasten von Patienten mit pulmonaler Hypertonie konnte durch die experimentelle Überexpression von miR-124 gezeigt werden, dass MCP-1 (monocyte chemoattractive protein-1), ein wichtiges Chemokine für mononukleäre Zellen, durch eine direkte Interaktion mit dem Zielgen in verminderten Konzentrationen gebildet wird. Umgekehrt konnte durch die Antagonisierung von miR-124 eine Erhöhung von MCP-1 erreicht werden: Die miR124 scheint demnach entscheidend für die perivaskuläre Inflammation bei der pulmonalen Hypertonie verantwortlich zu sein. In eleganten Folgeexperimenten zeigten die Autoren zudem, dass die Expression der miR-124 in Fibroblasten durch epigenetische Mechanismen (in diesem Fall durch acetylierende Modifikationen von Histonen) kontrolliert wird, was gleichzeitig die Möglichkeit für therapeutische Interventionen bieten könnte [46].

\section{$\operatorname{miR}-125$}

$\nabla$

miR-17 und miR-20 sind, wie oben erwähnt, antagonisierbar, sodass der pulmonale Gefäßumbau verhindert oder - wenn bereits etabliert - im Sinne eines „reverse remodellings“ verbessert werden kann. Allerdings sind beide miR, zumindest was das Hypoxie-Modell betrifft, unter $10 \%$ Sauerstoff nicht signifikant erhöht, weshalb die Suche nach weiteren, kausalen miR weitergeht. Als interessanter Kandidat konnte die miR-125 identifiziert werden, welche ebenfalls BMPR2 als Zielgen hat. Präliminäre Daten haben gezeigt, dass der Serumspiegel der miR-125 negativ mit dem pulmonal arteriellen Mitteldruck korreliert, d.h., je höher der pulmonale Mitteldruck ist, desto weniger miR ist im Serum vorhanden. Im experimentellen Modell der Hypoxie-induzierten pulmonalen Hypertonie wurden in hypoxischen Tieren (welche eine pulmonale Hypertonie entwickeln) ebenfalls verminderte Werte zirkulierender miR-125 gemessen, im Lungengewebe fanden sich allerdings erhöhte Werte. Die tiefen Serumwerte könnten somit Ausdruck des erhöhten intrazellulären Umsatzes sein, alternativ kann der verminderte Export durch die bei der pulmonalen Hypertonie bekanntermaßen gestörte Funktion von Dicer [35] bedingt sein. Ob die miR-125 ein interessanter Kandidat für einen pathogenetisch relevanten Biomarker im Serum von Patienten mit pulmonaler Hypertonie wird, muss allerdings in größeren, prospektiven Studien bestätigt werden [47].

\section{$\operatorname{miR}-145$}

$\nabla$

Cheng et al. zeigten 2009, dass die miR-145 in der Media der Gefäßwand übermäßig exprimiert ist, sie wird deshalb auch als phänotypischer Marker und Modulator der glatten Muskulatur betrachtet [48]. Die miR-145 scheint auch in der Entwicklung einer pulmonalen Hypertonie eine wichtige Rolle zu spielen. So hat die Gruppe um Caruso gezeigt, dass sich bei Wildtyp- Mäusen unter hypoxischen Bedingungen die Expression von miR-145 deutlich erhöht [35]. In miR-145-Knockout-Mäusen und in antimiR behandelten Mäusen kam es, gemessen anhand des systolischen rechtsventrikulären Druckes, der rechtsventrikulären Hypertrophie und dem Anteil des pulmonalen Gefäßumbaus, nicht zur Entwicklung einer Hypoxie-induzierten pulmonalen Hypertonie. Eine Defizienz von miR-145, aber auch eine Reduktion der miR-145 via anti-miR-Behandlung scheint somit einen protektiven Effekt zu haben. Zudem wurde im Lungengewebe von Patienten mit PAH eine deutliche Hochregulierung von miR-145 festgestellt, vermutlich über eine Mutation in BMPR2. Ob die Überexpression von miR-145 auch in der Hypoxie-induzierten pulmonalen Hypertonie über den BMPR2 zustande kommt (z.B. über die in der Hypoxie beschriebene Dysregulation von BMPR2), ist unklar [49].

\section{miR-150 \\ $\nabla$}

Ein Screening der gesamten im Plasma von Patienten mit PAH vorhandenen RNA zeigte, dass vor allem die miR-150 weniger stark exprimiert ist. Diese Beobachtung konnte in zwei verschiedenen Kohortenstudien gemacht werden. Die miR-150 scheint ein sehr guter Prädiktor des Krankheitsverlaufs zu sein, da die miR-150-Werte im Plasma signifikant mit dem 2-Jahres-Überleben korrelieren [50]. Je weniger zirkulierende miR-150 im Plasma vorhanden ist, desto geringer ist somit die Überlebenswahrscheinlichkeit des Patienten. Wieso die Menge an zirkulierender miR-150 bei Patienten mit PAH vermindert ist, bleibt noch unklar, ebenso die Frage, aus welchen Zellen die miR-150 freigesetzt wird.

\section{miR-204 \\ $\nabla$}

Eine weitere, gut untersuchte miR ist die miR-204. Sie wurde im Tierexperiment und im Gewebe von PAH-Patienten reduziert gefunden und konnte zudem mit dem Schweregrad der pulmonalen Hypertonie assoziiert werden. Ihre verminderte Expression führt zu einem Überhang des Signalweges src-STAT3-NFAT, wobei die beiden letzteren wiederholt im Zusammenhang mit der Pathogenese des pulmonalen Gefäßumbaus beschrieben wurden. Damit scheint es zu einem pro-proliferativen, anti-apoptotischen Phänotyp der glatten Gefäßmuskelzellen zu kommen. Die miR-204 wirkt auf die Regulation des STAT3-miR-17/-92 Signalweges und könnte diesen zumindest hypothetisch verstärken. Zudem hat die aerosolisierte intratracheale Verabreichung von miR-204 im Tiermodell zu einer signifikanten Abnahme des Remodellings und zu einer Normalisierung der pulmonalen Hämodynamik geführt. Sie stellt damit eine wichtige, potenziell kausaltherapeutische Option für Patienten mit pulmonaler Hypertonie dar [51]. 


\section{miR-424 und miR-503}

Ein wichtiger Signalweg in der Steuerung des vaskulären Remodellings involviert Apelin, welches an einen G-Protein-gekoppelten Rezeptor auf Gefäßendothelien bindet und unter anderem die endotheliale Stickstoffmonoxid-Synthese ankurbelt. In Patienten mit pulmonaler Hypertonie sind verminderte ApelinSerumwerte beschrieben, und Apelin-Knockout-Mäuse zeigen in hypoxischen Bedingungen eine stärkere Zunahme der pulmonalen Hypertonie als Wildtyp-Kontrollen [52]. Eine kürzlich publizierte Studie konnte zudem zeigen, dass in pulmonal-arteriellen Endothelzellen von PAH-Patienten der Apelin-Signalweg aufgrund von vermindert exprimierten miRs, nämlich miR-424 und miR-503, gestört ist. Dies wiederum führt zu einer verstärkten Reaktion von Wachstumsfaktoren (fibroblast growth factor FGF2) und vermehrter Proliferation von Gefäßzellen. Die therapeutische Verabreichung von miR-424 und miR-503 reduzierte im experimentellen Modell das vaskuläre Remodelling und bietet somit ebenfalls einen interessanten kausalen Therapieansatz [53].

\section{miR-22, miR-30, let-7, miR-322 und miR-451}

In den Tiermodellen von Caruso und Kollegen wurden folgende miRs signifikant verändert vorgefunden: miR-322, miR-451, miR-22, miR-30 und let-7 [35]. Dabei waren die miR-451 und 322 deutlich verstärkt vorhanden, die miR-322 allerdings fiel nach 21 Tagen unter hypoxischen Konditionen bzw. nach Monocrotalin-Injektion wieder auf das Level der Kontrollgruppe. Die miR-22, 30c, let-7a und let-7f wurden als signifikant vermindert gemessen, auch hier zeigten sich allerdings deutliche Unterschiede je nach Zeitpunkt und experimentellem Modell.

Diese Unterschiede widerspiegeln vermutlich die pathophysiologische Art, wie beide Modelle eine pulmonale Hypertonie induzieren: Die Hypoxie führt primär zu einer Vasokonstriktion, sie stimuliert zudem Veränderungen in allen Gefäßschichten, führt im Unterschied zur Monocrotalin-Injektion allerdings kaum zu einer Entzündungsantwort.

Die bioinformatische Analyse, ebenfalls von Caruso und Kollegen durchgeführt, wies darauf hin, dass vor allem die in diesem Abschnitt erwähnten miRs mehrere gemeinsame Zielgene haben [35]. Diese Eigenschaft der miR weist auf die komplexe Regulierung von solchen „targets“ hin.

\section{Therapie/Perspektive}

Schweregrad, Prognose und Chronizität der pulmonalen Hypertonie werden über das pulmonal-vaskuläre Remodelling definiert. Die heute verwendeten spezifischen Vasodilatatoren haben zwar Lebensqualität und Mortalität der $\mathrm{P}(\mathrm{A}) \mathrm{H}$-Patienten enorm verbessert, die pulmonale Hypertonie bleibt aber eine schwere, unheilbare Erkrankung ohne kausale Therapie. Der Off-LabelEinsatz von Tyrosinkinaseinhibitoren (Imatinib) hat in Fallbeschreibungen zwar zu einer Abnahme des pulmonalen Mitteldrucks und im experimentellen Modell zu einem „reverse Remodelling“ geführt, die Euphorie wurde in der IMPRES-Studie allerdings durch das Auftreten schwerwiegender unerwünschter Wirkungen (Subduralhämatome) gedämpft [54]; beim Einsatz eines anderen Tyrosinkinasehemmers (Dasatinib) wurde sogar die Entstehung einer pulmonalen Hypertonie beschrieben [55]. Aufgrund der Komplexität der multiplen Tyrosinkinasen ist der ideale Hemmer zur Behandlung der pulmonalen Hypertonie aktuell nicht vorhanden. Es bleibt auch unklar, ob miRs, welche die Expression dieser Tyrosinkinase-Rezeptoren (z.B. PDGF-R) steuern, hier eine therapeutische Rolle spielen können.

Neben ihrem Effekt auf den Gefäßumbau wirken miRs - indirekt oder direkt - auch auf die Kardiomyozyten des rechten Ventrikels. Die meisten experimentellen Studien haben zwar nicht untersucht, ob die Veränderungen der rechtsventrikulären Hypertrophie direkt durch AntagomiRs oder die Überexpression von miRs verursacht werden. Die miR-21 wurde aber wiederholt mit der Entstehung einer kardialen Fibrose $[56,57]$ und der rechtsventrikulären Funktion [58] beschrieben und könnte in diesem Zusammenhang als rechtsventrikuläre „PH-targeted“-Therapie interessant werden.

AntagomiRs sind spezifische Antagonisten von miRs [59], sie werden als komplementäre Sequenz designed und zur Erhöhung der biologischen Stabilität mit einem Cholesterol-Ester oder einem verstärkten „backbone“ (LNA) modifiziert. AntagomiRs können intravenös, intraperitoneal oder per Inhalation appliziert werden und weisen kaum einen hepatischen First-Pass-Effekt auf, ihre Halbwertszeit scheint mehrere Tage zu betragen. Im Vergleich zu siRNA sind AntagomiRs kürzer und damit spezifischer, auch sind ihre Nebenwirkungen geringer. Der Einsatz von AntagomiRs scheint im Tiermodell gut tolerabel zu sein (gemessen am Verhalten der Tiere sowie an der hepatischen Interferon-Antwort als Surrogatmarker); auch in klinischen Studien bei Hepatitis C-Patienten, bei denen die miR-122 gehemmt wird, ist die Verträglichkeit gut $[60,61]$. Ein mögliches Problem stellen „off-target“-Effekte dar, d.h. Medikamentenwirkung außerhalb des gewünschten Zielorgans. Um den Wirkstoff vorwiegend in der Lunge zu applizieren, bietet die Verabreichung per Inhalation eine elegante Methode, welche zumindest im Tiermodell bereits erfolgreich angewendet wurde [51]. Diese präliminären Daten machen miRs zu einem attraktiven therapeutischen „target“ und AntagomiRs zu einem potenziell kausalen Therapieansatz in der Behandlung des Gefäßumbaus bei der pulmonalen Hypertonie.

\section{Interessenkonflikt}

$\nabla$

Das Projekt „the role of microRNAs in pulmonary hypertension: diagnosis and treatment" wird vom Schweizerischen Nationalfonds unterstützt (SNF Grant 31003A_144212).

\section{Literatur}

1 Simonneau G, Galie N, Rubin LJ et al. Clinical classification of pulmonary hypertension. J Am Coll Cardiol 2004; 43: 5-12

2 Chemla D, Castelain V, Humbert $M$ et al. New formula for predicting mean pulmonary artery pressure using systolic pulmonary artery pressure. Chest 2004; 126: 1313-1317

3 Rich JD, Shah SJ, Swamy RS et al. Inaccuracy of Doppler echocardiographic estimates of pulmonary artery pressures in patients with pulmonary hypertension: implications for clinical practice. Chest 2011; 139: 988-993

4 Galie N, Torbicki A, Barst R et al. Guidelines on diagnosis and treatment of pulmonary arterial hypertension. The Task Force on Diagnosis and Treatment of Pulmonary Arterial Hypertension of the European Society of Cardiology. Eur Heart J 2004; 25: 2243 - 2278

5 Opitz CF, Blindt R, Blumberg $F$ et al. Pulmonary hypertension: hemodynamic evaluation: hemodynamic evaluation - recommendations of the Cologne Consensus Conference 2010. Dtsch Med Wochenschr $2010 ; 135: 78-86$ 
6 Machado RF, Londhe Nerkar MV, Dweik RA et al. Nitric oxide and pulmonary arterial pressures in pulmonary hypertension. Free radical biology \& medicine 2004; 37: 1010 - 1017

7 Pietra GG, Capron F, Stewart S et al. Pathologic assessment of vasculopathies in pulmonary hypertension. J Am Coll Cardiol 2004; 43: 25S$32 \mathrm{~S}$

8 Morrell NW. Screening for pulmonary arteriovenous malformations. Am J Respir Crit Care Med 2004; 169: 978-979

9 Olschewski H, Seeger $W$. Treatment of pulmonary artery hypertension. Pneumologie 2000; 54: 06222-224

10 Pietra GG, Capron F, Stewart $S$ et al. Pathologic assessment of vasculopathies in pulmonary hypertension. J Am Coll Cardiol 2004; 43: 25 - 32

11 Dorfmuller P, Humbert M, Capron F. Update on the pathomorphological assessment of vasculopathies in pulmonary arterial hypertension. Pathologe 2006; 27: 140-146

12 Hassoun PM, Krishnan JA. Pulmonary hypertension as a risk factor for death in patients with sickle cell disease. N Engl J Med 2004; 350: $2521-2522$, author reply 2521-2522

13 Humbert M, Monti G, Brenot $F$ et al. Increased interleukin-1 and interleukin-6 serum concentrations in severe primary pulmonary hypertension. Am J Respir Crit Care Med 1995; 151: 1628-1631

14 Soon E, Holmes AM, Treacy CM et al. Elevated levels of inflammatory cytokines predict survival in idiopathic and familial pulmonary arterial hypertension. Circulation 2010; 122: 920-927

15 Ulrich S, Taraseviciene-Stewart L, Huber LC et al. Peripheral blood B lymphocytes derived from patients with idiopathic pulmonary arterial hypertension express a different RNA pattern compared with healthy controls: a cross sectional study. Respir Res 2008; 9: 20

16 Balabanian K, Foussat A, Dorfmuller P et al. CX(3)C chemokine fractalkine in pulmonary arterial hypertension. Am J Respir Crit Care Med 2002; 165: 1419-1425

17 Dorfmuller P, Zarka V, Durand-Gasselin I et al. Chemokine RANTES in severe pulmonary arterial hypertension. Am J Respir Crit Care Med 2002; 165: $534-539$

18 Perros F, Dorfmuller P, Souza R et al. Fractalkine-induced smooth muscle cell proliferation in pulmonary hypertension. Eur Respir J 2007; 29: 937-943

19 Schermuly RT, Dony E, Ghofrani HA et al. Reversal of experimental pulmonary hypertension by PDGF inhibition. J Clin Invest 2005; 115: $2811-2821$

20 Bonnet S, Rochefort G, Sutendra G et al. The nuclear factor of activated T cells in pulmonary arterial hypertension can be therapeutically targeted. Proc Natl Acad Sci U S A 2007; 104: 11418 - 11423

21 Weir EK, Olschewski AK. Role of ion channels in acute and chronic responses of the pulmonary vasculature to hypoxia. Cardiovasc Res 2006; 71: 630-641

22 Marcos E, Adnot S, Pham MH et al. Serotonin transporter inhibitors protect against hypoxic pulmonary hypertension. Am J Respir Crit Care Med 2003; 168: 487-493

23 Ulrich S, Szamalek-Hoegel J, Hersberger $M$ et al. Sequence variants in BMPR2 and genes involved in the serotonin and nitric oxide pathways in idiopathic pulmonary arterial hypertension and chronic thromboembolic pulmonary hypertension: relation to clinical parameters and comparison with left heart disease. Respiration 2010; 79: 279-287

24 Machado RD, Eickelberg O, Elliott CG et al. Genetics and genomics of pulmonary arterial hypertension. J Am Coll Cardiol 2009; 54: 0132 - 42

25 Lane KB, Machado RD et al. International PPHC. Heterozygous germline mutations in BMPR2, encoding a TGF-beta receptor, cause familial primary pulmonary hypertension. Nat Genet 2000; 26: 81-84

26 Sztrymf B, Coulet F, Girerd B et al. Clinical outcomes of pulmonary arterial hypertension in carriers of BMPR2 mutation. Am J Respir Crit Care Med 2008; 177: 1377-1383

27 Montani D, Achouh L, Dorfmuller P et al. Pulmonary veno-occlusive disease: clinical, functional, radiologic, and hemodynamic characteristics and outcome of 24 cases confirmed by histology. Medicine (Baltimore) 2008; 87: 220-233

28 Atkinson C, Stewart S, Upton PD et al. Primary pulmonary hypertension is associated with reduced pulmonary vascular expression of type II bone morphogenetic protein receptor. Circulation 2002; 105: 1672 1678

29 Ishida $K$, Masuda M, Tanabe $N$ et al. Long-term outcome after pulmonary endarterectomy for chronic thromboembolic pulmonary hypertension. J Thorac Cardiovasc Surg 2012; 144: 321 - 326

30 Morty RE, Nejman B, Kwapiszewska G et al. Dysregulated bone morphogenetic protein signaling in monocrotaline-induced pulmonary ar- terial hypertension. Arteriosclerosis, thrombosis, and vascular biology 2007; 27: 1072-1078

31 Takahashi H, Goto N, Kojima Y et al. Downregulation of type II bone morphogenetic protein receptor in hypoxic pulmonary hypertension. Am J Physiol Lung Cell Mol Physiol 2006; 290: 450-458

32 Brock M, Trenkmann M, Gay RE et al. Interleukin-6 modulates the expression of the bone morphogenic protein receptor type II through a novel STAT3-microRNA cluster 17/92 pathway. Circulation research 2009; 104: 1184-1191

33 Dewachter L, Adnot S, Guignabert C et al. Bone morphogenetic protein signalling in heritable versus idiopathic pulmonary hypertension. Eur Respir J 2009; 34: 1100 - 1110

34 Long L, Crosby A, Yang X et al. Altered bone morphogenetic protein and transforming growth factor-beta signaling in rat models of pulmonary hypertension: potential for activin receptor-like kinase-5 inhibition in prevention and progression of disease. Circulation 2009; 119: $566-$ 576

35 Caruso P, Maclean MR, Khanin $R$ et al. Dynamic changes in lung microRNA profiles during the development of pulmonary hypertension due to chronic hypoxia and monocrotaline. Arterioscler Thromb Vasc Biol 2010; 30: 716-723

36 Brock $M$, Trenkmann $M$, Gay RE et al. MicroRNA-18a enhances the interleukin-6-mediated production of the acute-phase proteins fibrinogen and haptoglobin in human hepatocytes. J Biol Chem 2011; 286: 40142 - 40150

37 Kosaka N, Ochiya T. Unraveling the Mystery of Cancer by Secretory microRNA: Horizontal microRNA Transfer between Living Cells. Front Genet 2011; 2: 97

38 Kosaka N, Takeshita F, Yoshioka $Y$ et al. Exosomal tumor-suppressive microRNAs as novel cancer therapy: "exocure" is another choice for cancer treatment. Adv Drug Deliv Rev 2013; 65: 376- 382

39 Masri FA, Xu W, Comhair SA et al. Hyperproliferative apoptosis-resistant endothelial cells in idiopathic pulmonary arterial hypertension. Am J Physiol Lung Cell Mol Physiol 2007; 293: 548-554

40 Pullamsetti SS, Doebele C, Fischer A et al. Inhibition of MicroRNA-17 Improves Lung and Heart Function in Experimental Pulmonary Hypertension. Am J Respir Crit Care Med 2012; 185: 409-419

41 Brock M, Samillan VJ, Trenkmann $M$ et al. AntagomiR directed against miR-20a restores functional BMPR2 signalling and prevents vascular remodelling in hypoxia-induced pulmonary hypertension. European heart journal 2012; DOI 10.1093/eurheart/ehs060 [electronic publication only]

42 Yang S, Banerjee S, Freitas A et al. miR-21 regulates chronic hypoxia-induced pulmonary vascular remodeling. Am J Physiol Lung Cell Mol Physiol 2012; 302: 521 - 529

43 Parikh VN, Jin RC, Rabello $S$ et al. MicroRNA-21 integrates pathogenic signaling to control pulmonary hypertension: results of a network bioinformatics approach. Circulation 2012; 125: 1520-1532

44 Kang $K$, Peng X, Zhang $X$ et al. MicroRNA-124 suppresses the transactivation of nuclear factor of activated $\mathrm{T}$ cells by targeting multiple genes and inhibits the proliferation of pulmonary artery smooth muscle cells. J Biol Chem 2013; 288: 25414-25427

45 Wang $D$, Zhang $H$, Li M et al. MicroRNA-124 controls the proliferative, migratory, and inflammatory phenotype of pulmonary vascular fibroblasts. Circ Res 2014; 114: 67-78

46 Pohl NM, Fernandez RA, Smith KA et al. Deacetylation of MicroRNA-124 in fibroblasts: role in pulmonary hypertension. Circ Res 2014; 114: 5 8

47 Huber L, Ulrich S, Gassmann M et al. Serum levels of microRNA-125a are decreased in patients with precapillary pulmonary hypertension and correlate inversely with hemodynamics. ERS Annual Congress 2013; Abstract Number 3416

48 Cheng Y, Liu X, Yang J et al. MicroRNA-145, a novel smooth muscle cell phenotypic marker and modulator, controls vascular neointimal lesion formation. Circ Res 2009; 105: 158-166

49 Caruso P, Dempsie Y, Stevens HC et al. A role for miR-145 in pulmonary arterial hypertension: evidence from mouse models and patient samples. Circ Res 2012; 111: 290 - 300

50 Rhodes CJ, Wharton J, Boon RA et al. Reduced microRNA-150 is associated with poor survival in pulmonary arterial hypertension. Am J Respir Crit Care Med 2013; 187: 294-302

51 Courboulin A, Paulin R, Giguere NJ et al. Role for miR-204 in human pulmonary arterial hypertension. J Exp Med 2011; 208: 535-548 
52 Chandra SM, Razavi H, Kim J et al. Disruption of the apelin-APJ system worsens hypoxia-induced pulmonary hypertension. Arterioscler Thromb Vasc Biol 2011; 31: 814-820

53 Kim J, Kang Y, Kojima Y et al. An endothelial apelin-FGF link mediated by miR-424 and miR-503 is disrupted in pulmonary arterial hypertension. Nat Med 2013; 19: 74-82

54 Hoeper MM, Barst RJ, Bourge RC et al. Imatinib mesylate as add-on therapy for pulmonary arterial hypertension: results of the randomized IMPRES study. Circulation 2013; 127: 1128-1138

55 Montani D, Bergot E, Gunther $S$ et al. Pulmonary arterial hypertension in patients treated by dasatinib. Circulation 2012; 125: 2128-2137

56 Thum T, Gross C, Fiedler J et al. MicroRNA-21 contributes to myocardial disease by stimulating MAP kinase signalling in fibroblasts. Nature 2008; 456: 980-984

57 Dong S, Ma W, Hao B et al. microRNA-21 promotes cardiac fibrosis and development of heart failure with preserved left ventricular ejection fraction by up-regulating Bcl-2. Int J Clin Exp Pathol 2014; 7: 565 - 574

58 Liu $F$, Yin $L$, Zhang $L$ et al. Trimetazidine improves right ventricular function by increasing miR-21 expression. Int J Mol Med 2012; 30: $849-855$
59 Krutzfeldt J, Rajewsky N, Braich $R$ et al. Silencing of microRNAs in vivo with 'antagomirs'. Nature 2005; 438: 685-689

60 Janssen HL, Reesink HW, Lawitz EJ et al. Treatment of HCV infection by targeting microRNA. N Engl J Med 2013; 368: 1685-1694

61 Lieberman J, Sarnow P. Micromanaging hepatitis C virus. N Engl J Med 2013; 368: $1741-1743$

62 Brock M, Gassmann M, Speich $R$ et al. The hypoxia-induced miR-130 increases proliferation of pulmonary arterial smooth muscle cells by targeting the tumor suppressor CDKN1A (p21). ERS Annual Congress 2013; Abstract Number 1500

63 Rhodes CJ, Wharton J, Boon RA et al. Reduced miR-150 is associated with poor survival in pulmonary arterial hypertension. Am J Respir Crit Care Med 2012; 187: 294-302

64 Winter J, Jung S, Keller $S$ et al. Many roads to maturity: microRNA biogenesis pathways and their regulation. Nat Cell Biol 2009; 11: $228-$ 234

65 Brock M, Huber LC. Vascular remodelling in hypoxia-induced pulmonary hypertension: role of cytokines and microRNAs. PVRI Review 2013; 5: 20-23 